\title{
Structural Characterization of Li-excess Cathode Materials for Lithium-Ion Batteries
}

\author{
K.A. Jarvis, ${ }^{*}$ Z.Q. Deng, ${ }^{*}$, L. F. Allard,** A. Manthiram, ${ }^{*}$ and P.J. Ferreira* \\ * Materials Science and Engineering Program, University of Texas at Austin, Austin, Texas 78712. \\ ** Materials Science \& Tech. Div, Oak Ridge National Laboratory, Oak Ridge, Tennessee 37831
}

Among the most promising cathode materials for Li-ion batteries are Li-excess oxides of the type $\mathrm{Li}\left[\mathrm{Li}_{\mathrm{x}} \mathrm{M}_{1-\mathrm{x}}\right] \mathrm{O}_{2}(\mathrm{M}=\mathrm{Mn}, \mathrm{Co}, \mathrm{Ni})$, which show a significantly higher capacity $(>250 \mathrm{mAh} / \mathrm{g})$ compared with commercial $\mathrm{LiCoO}_{2}$ cathodes. However, the structure of these materials and the effect of structure on the overall capacity have been the subject of much debate. Some groups have reported these lithium-excess oxides to be a solid solution, in which the $\mathrm{Li}^{+}$ions order but the transition metal ions are randomly distributed [1], while others have reported that these materials are composed of ordered domains of $\mathrm{Li}_{2} \mathrm{MnO}_{3}$ integrated within a disordered $\mathrm{LiMO}_{2}$ structure [2,3]. To address this issue, we have used aberration-corrected STEM imaging and computer simulations, as well as a novel STEM electron diffraction technique, called D-STEM [4], to study the lithium-excess compound $\mathrm{Li}\left[\mathrm{Li}_{.2} \mathrm{Mn}_{.6} \mathrm{Ni}_{.2}\right] \mathrm{O}_{2}$.

The $\mathrm{Li}\left[\mathrm{Li}_{\mathrm{x}} \mathrm{M}_{1-\mathrm{x}}\right] \mathrm{O}_{2}$ sample was first observed in a JEOL 2200FS STEM/TEM fitted with a CEOS $\mathrm{GmbH}$ corrector on the illuminating lenses, and equipped with a high-angle annular dark-field (HAADF) detector. STEM images were simulated with the HREM Simulation Suite, which is based on the FFT Multislice technique [5]. The samples were also characterized by the D-STEM technique [4], using a JEOL 2010F. To assist in the interpretation of the STEM images, STEM simulations were considered for the solid solution case and the two-phase system. To perform the simulations, the structures of $\mathrm{Li}_{2} \mathrm{MnO}_{3}$ and $\mathrm{LiMO}_{2}$ were taken from literature [6-7]. The structure of the solid solution $\mathrm{Li}\left[\mathrm{Li}_{.2} \mathrm{Mn}_{.6} \mathrm{Ni}_{2}\right] \mathrm{O}_{2}$, was determined from XRD data and XRD simulations, and found to have an ordering of $\mathrm{Li}^{+}$and $\mathrm{Mn}^{4+}$ ions in the transition metal layer with the $\mathrm{Mn}^{4+}$ ions randomly replaced by $\mathrm{Ni}^{2+}$ ions. Figure 1 a shows the two-phase case containing both regions of continuous bright columns (disordered regions), and regions of two bright columns followed by a dark column (ordered regions). For the solid solution case, the materials should be uniform throughout, where two bright columns are followed by a less bright column (Fig. 1b). According to the actual STEM image shown in Figure 2, the best match between experiment and simulation occurs for the solid solution case. Yet, the electron diffraction (ED) pattern corresponding to the solid solution structure (Fig. 3) does not contain only single spots, as would be expected for a defect-free single phase. Instead, it exhibits multiple spots and streaks. A careful examination of the STEM image reveals planar defects on the transition metal layer, which result in rotations of the crystal lattice. Thus, the multiple spots and streaks in the ED pattern are related to Li-ordering in the transition metal layer and consequent presence of planar defects.

To verify these results, further analysis from multiple particles in multiple areas was needed, including thicker regions where aberration-corrected STEM imaging cannot resolve the structure. DSTEM uses a small probe $(\sim 1 \mathrm{~nm})$ that can be scanned across a defined location to produce electron diffraction patterns from spots, lines, or areas. This technique was employed to determine if any disordered regions existed. No disordered regions were found in any of the multiple particles 
analyzed. Thus, the D-STEM results confirm the STEM results, namely that the $\mathrm{Li}\left[\mathrm{Li}_{.2} \mathrm{Mn}_{.6} \mathrm{Ni}_{.2}\right] \mathrm{O}_{2}$ material is a solid solution with Li-ordering and multiple planar defects.

References

[1] Z. Lu, Z. Chen and J. R. Dahn, Chem. Mater. 15 (2003), 15, 3214.

[2] M. M. Thackeray et al., J. Mater. Chem. 30 (2007), 3053.

[3] M. M. Thackeray, J. Chem. Eng. Jpn. 40 (2007), 1150.

[4] K. J. Ganesh et al., Microsc. Microanal. 16 (2010), 614.

[5] K. Ishizuka, Ultramicroscopy 90 (2001), 71.

[6] Y. S. Meng et al., Chem. Mater. 17 (2005), 2386.

[7] P. Strobel et al., J. Solid State Chem. 75 (1988), 90.

[8] This work was supported in part by the Department of Energy, Office of Basic Energy Sciences as part of the Energy Frontier Research Center (EFRC); microscopy at Oak Ridge National Laboratory was sponsored by the U. S. Department of Energy, Office of Energy Efficiency and Renewable Energy, Vehicle Technologies Program.

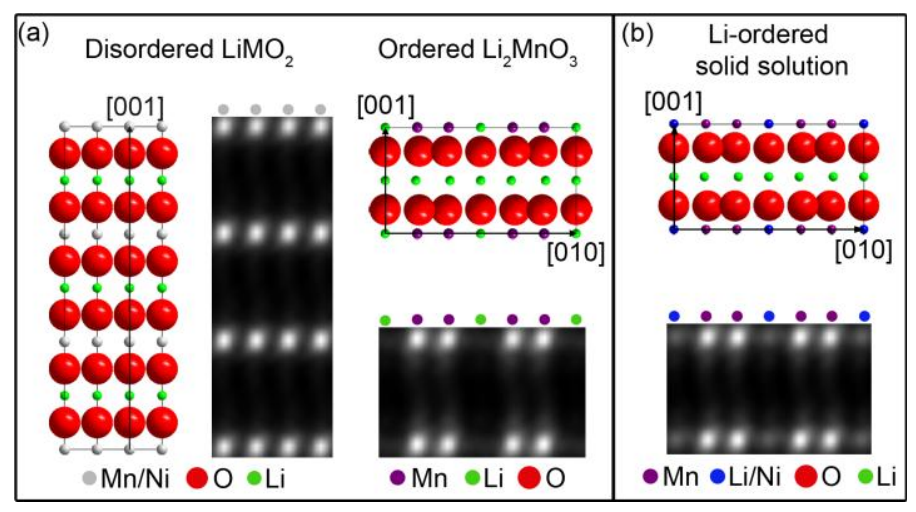

FIG.1 Schematics of the unit cells and corresponding STEM simulations for (a) a two-phase material with regions of $\mathrm{LiMO}_{2}$ and $\mathrm{Li}_{2} \mathrm{MnO}_{3}$ and (b) a solid solution with Li-ordering. Each crystal is rotated such that the TM planes are parallel to the beam and alike atoms are stacked on top of one

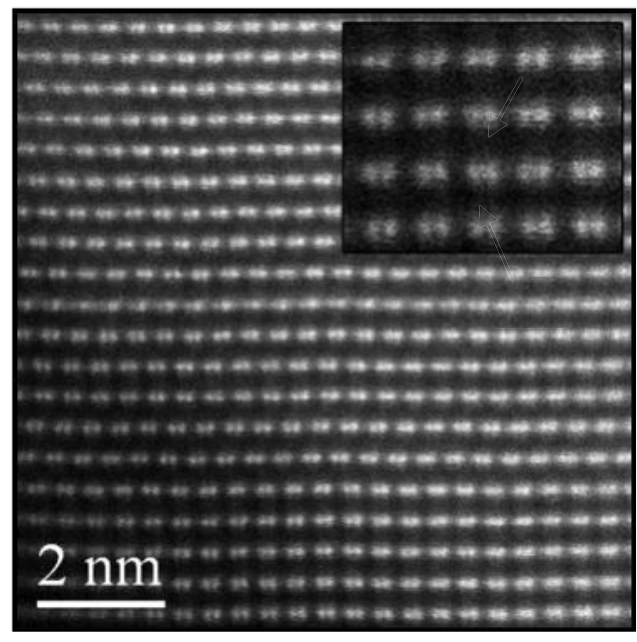

FIG. 2. Aberration-corrected STEM image of a $\mathrm{Li}\left[\mathrm{Li}_{0.2} \mathrm{Ni}_{0.2} \mathrm{Mn}_{0.6}\right] \mathrm{O}_{2}$ rotated such that the $\mathrm{TM}$ planes are parallel to the beam and alike atoms are stacked on top of one another.

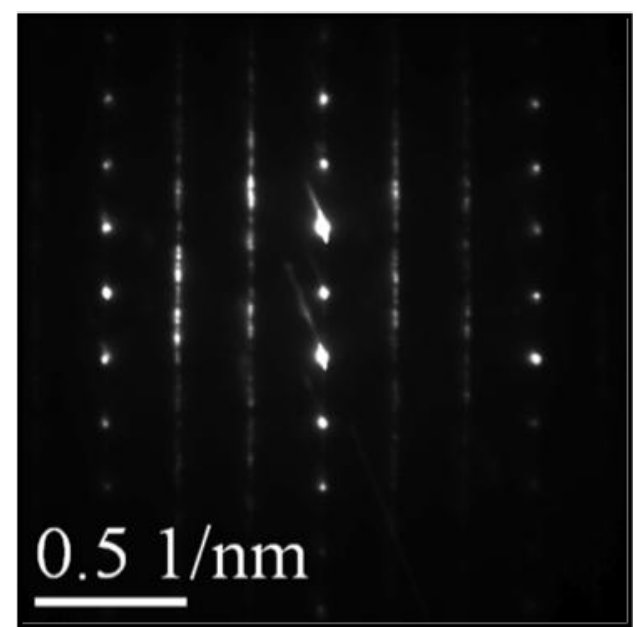

FIG. 3. ED pattern of a $\mathrm{Li}\left[\mathrm{Li}_{0.2} \mathrm{Ni}_{0.2} \mathrm{Mn}_{0.6}\right] \mathrm{O}_{2}$ crystal rotated such that the TM planes are parallel to the beam and alike atoms are stacked on top of one another. 